\title{
Новикова Е.В.
}

Московский научно-практический центр медицинской реабилитации, восстановительной и спортивной медицины Департамента здравоохранения г. Москвы; 105120, г. Москва, д. 53

\section{ЛЕЧЕНИЕ И МЕДИЦИНСКАЯ РЕАБИЛИТАЦИЯ ДЕТЕЙ С ГИПЕРАКТИВНЫМ МОЧЕВЫМ ПУЗЫРЕМ}

Для корреспонденции: Новикова Е.В., 105120, г. Москва, Земляной вал, д.53; тел. +7(916)758-75-61, E-Mail: minkamal@mail.ru Для цитИРОВанИЯ: НОВИКОВа Е.В. ЛЕЧЕНИЕ И МЕДИЦИНСКАЯ РЕАБИЛИТАЦИЯ ДЕТЕЙ С ГИПЕРАКТИВНЫМ МОЧЕВЫМ ПУЗЫРЕМ Российский вестник детской хирургии, анестезиологии и реаниматологии; 2018; 8(4): 105-110 https://doi.org/10.30946/2219-4061-2018-8-4-105-110

Получена: 03.09.2018. Принята к печати: 23.11.2018 Информация о финансировании и конффликте интересов

Авторы декларируют отсутствие явных и потенциальных конфликтов интересов, связанных с публикацией настоящей статьи. Источник финансирования не указан.

\section{Резюме}

В статье представлены современные технологии медицинской реабилитации детей с гиперактивным мочевым пузырем (ГАМП), направленные на совершенствование акта мочеиспускания на всех уровнях иннервации мочевого пузыря. Медицинская реабилитация детей с ГАМП включает в себя поведенческую терапию и медикаментозное лечение. Популярность физиотерапевтических методов объясняется влиянием на основные звенья патогенеза, отсутствием побочных эффектов и возможностью применения у детей раннего возраста. В комплексном лечении нейрогенной дисфункции мочевого пузыря используют широкий спектр методов физиотерапии, действие которых направлено на регуляцию акта мочеиспускания на уровне супраспинальных и спинальных центров мочеиспускания, систему детрузор-стабилизирующих рефлексов, а также на улучшение кровообращения и обменно-трофических процессов в детрузоре. Сегодня естественные и преформированные методы физиотерапии применяют в комплексе с лечебной физкультурой, массажем, БОС-терапией.

Ключевые слова: нейрогенная дисфункиия мочевого пузыря, медицинская реабилитачия, гиперактивный мочевой пузырь, интерференционные токи, дэнс, электросон, эпс, бос-терапия, лфк, массаж, дети 


\section{Elena V. Novikova}

Moscow Research Center for Medical Rehabilitation, Restorative and Sports Medicine of Moscow Health Department; Zemlyanoi val, 53, Moscow, Russia, 105120

\section{TREATMENT AND MEDICAL REHABILITATION IN CHILDREN WITH A HYPERACTIVE BLADDER}

For correspondence: Elena V. Novikova, Earthworks street, 53, Moscow, Russia, 105120; tel. +7(916)758-75-61; E-Mail: minkamal@mail.ru For citation: Elena V. Novikova. TREATMENT AND MEDICAL REHABILITATION IN CHILDREN WITH A HYPERACTIVE BLADDER Journal of Pediatric Surgery, Anesthesia and Intensive Care; 8(4): 105-110 https://doi.org/10.30946/2219-4061-2018-8-4-105-110

Received: 03.09.2018. Adopted for publication: 23.11.2018

Information on funding and conflict of interest

The authors declare the absence of obvious and potential conflicts of interest related to the publication of this article. Source of funding is not specified

\section{Abstract}

The article describes modern technologies of medical rehabilitation in children with a hyperactive bladder (HAB) aimed at the perfection of a urinary act at any levels of bladder innervation. Medical rehabilitation of children with HAB includes behavioral therapy and drug therapy. Physiotherapeutic methods are popular due to the effect on the basic links of pathogenesis, lack of adverse effects and possibility of use in infants. The complex treatment of neuro- genic bladder dysfunction uses a wide spectrum of physiotherapeutic methods aimed at the regulation of urination acts at the level of supraspinal and spinal urination centers, system of detrusor stabilizing reflexes and improvement of blood supply and metabolic trophic processes in the detrusor. Natural and preformed methods of physiotherapy are currently used along with exercise therapy, massage and biofeedback therapy.

Key words: neurogenic bladder dysfunction, medical rehabilitation, hyperactive urinary bladder, interferential currents, dens, electrosleep, elastic pseudoboiling layer, exercise therapy, massage, children

Актуальность и социальная значимость проблемы реабилитации детей с нейрогенной дисфункцией мочевого пузыря обусловлены высокой распространенностью, возможностью развития нарушений уродинамики, значительным снижением качества жизни $[1,2,3]$. Практически все дети с расстройствами мочеиспускания находятся в состоянии социальной дезадаптации. По данным официальной статистики, недержанием мочи страдают 10-30\% детей. Среди детей с сочетанной уронефрологической патологией недержание мочи встречается в $50-60 \%$ случаев [4].

Наиболее тяжелые расстройства функции мочевого пузыря наблюдаются при врожденных пороках развития (синдром миелодисплазии), травмах, опухолях и воспалительно-дегенеративных заболеваниях спинного мозга $[1,5]$.

Нарушение функции мочевого пузыря характерно для дтей с пороками развития мочевого пузыря и уретры - эписпадии, экстрофии мочевого пузыря, воспалительных заболеваний мочеполовых органов.

В педиатрической практике наиболее часто встречаются нейрогенные дисфункции мочевого пузыря (НДМП), обусловленные задержкой созревания высших центров вегетативной регуляции нижнего отдела мочевого тракта. Проявляясь клинически синдромом гиперактивного мочевого пузыря в виде поллакиурии, ургентных позывов, ургентного недержания, энуреза, они не только вызывают энурез, но и сопровождаются тяжелыми нарушениями уродинамики по функционально-обструктивному типу, участвуют в формировании цистита, пиелонефрита, пузырно-мочеточникового рефлюкса $[6,7]$.

Система медицинской реабилитации детей с нейрогенной дисфункцией мочевого пузыря имеет многоуровневый характер и проводится на стационарном, поликлиническом, санаторном этапах, в центрах (отделениях) медицинской реабилитации, дневных ста- 
ционарах. Медицинская реабилитация детей с нейрогенной дисфункцией мочевого пузыря включает спектр лекарственных и немедикаментозных технологий, таких как преформированные физические факторы, бальнеотерапия, теплолечение, ЛФК, массаж, рефлексотерапия, гомеопатия, БОС-терапия, уротерапия.

Согласно рекомендациям международной организации по проблемам недержания мочи у детей ICCS, лечение начинают с уротерапии - системы когнитивных методов, направленных на помощь семье в соблюдении режима дня, питьевого режима, объяснение ребенку и родителям, как правильно опорожнять мочевого пузыря и следить за частотой стула, вести дневник регистрации частоты мочеиспусканий $[6,8,9,10]$.

При неэффективности уротерапии назначают фармакотерапию.

Наиболее успешно у детей с ГАМП применяют антихолинергические средства или антагонисты М-холинорецепторов (дриптан, детрузитол, спазмекс, платифиллин), а также ноотропы и лекарственные препараты, обладающие антигипоксическим действием (препараты янтарной кислоты, коферментные формы витаминов группы B, L-карнитин) [11, 12, 13, 14].

В настоящее время разработаны нелекарственные технологии медицинской реабилитации детей с нейрогенной дисфункцией мочевого пузыря. На фоне медикаментозной коррекции или самостоятельно применяют методы физиотерапии, позволяющие воздействовать на различные патогенетические звенья заболевания, направленные на регуляцию акта мочеиспускания на всех уровнях иннервации мочевого пузыря $[12,15,16]$.

С целью воздействия на корковые и подкорковые центры мочеиспускания, применяют электросон, эндоназальный электрофорез витаминов группы В, гальванический воротник по Щербаку. Для расширения влияния на неврогенные механизмы нейрогенной дисфункции мочевого пузыря у детей применяют новый метод нейротропной импульсной электротерапии - ИНФИТА-терапию.

Эффективно при воздействии на спинальные центры мочеиспускания проведение ультразвука, импульсных токов. Среди методов импульсных воздействий одним из эффективных и безопасных методов является динамическая электронейростимуляция, преимуществом которой является возможность применения импульсов по форме и частоте сходных с потенциалами действия внутренних ор- ганов и наличие «биологической» обратной связи, позволяющей проводить диагностику перед процедурой $[16,17]$. Сегодня доказано благоприятное влияние динамической электронейростимуляции на клиническое течение и состояние уродинамики у детей с НДМП. По данным функционального исследования мочевого пузыря отмечалось увеличение эффективного объема мочевого пузыря, снижение внутрипузырного давления $[17,18]$.

Для оказания воздействия на систему детрузор-стабилизирующих рефлексов эффективно проведение анальной стимуляции синусоидальными модулированными токами, интерференционными токами. Интерференционные токи являются перспективным физическим фактором в детской урологии, обладают малой энергетической нагрузкой, мягким воздействием, возможностью целенаправленного воздействия на глубоко расположенные органы. Интерференционные токи (ИТ) проводятся по поперечно-диагональной методике с частотой 10-20 Гц, 8-10 мин; на курс лечения 10 ежедневных процедур. В последние годы разработаны дифференцированные технологии применения интерференционных токов в лечении НДМП с сопутствующим пиелонефритом. [19, 20].

В лечении ГАМП наиболее успешно применяют М-холиноблокаторы. Механизм действия антихолинергических препаратов заключается в блокаде М-холинорецепторов детрузора, что в свою очередь уменьшает действие ацетилхолина на детрузор, снижая его гиперактивность. Наряду с пероральным приемом М-холиноблокаторов у детей с ГАМП, применяют введение этих препаратов путем электрофореза, научно обоснованной методикой в педиатрии является применение атропинэлектрофореза на область мочевого пузыря $[6,11]$.

В медицинской реабилитации детей с НДМП применяют методы физиотерапии, направленные на улучшение трофики детрузора, устранение гипоксии и метаболических нарушений в стенке мочевого пузыря. Предпочтение отдают электрофорезу и фонофорезу лекарственных препаратов, улучшающих микроциркуляцию, таких, как эуфиллин, никотиновая кислота, препаратов лечебной грязи.

В настоящее время доказана эффективность лазерного излучения в медицинской реабилитации детей с ГАМП. Лазерное излучение проводится на область проекции мочевого пузыря и крестцовый отдел позвоночника инфракрасным лазером 
(0.89 мкм) в импульсном режиме. Мощность излучения составляет 2 Вт, частота излучения 80 Гц, длительность процедуры от 4 до 8 мин., на курс лечения 7-8 ежедневных процедур. Под влиянием лазерного излучения отмечается нормализация акта мочеиспускания с восстановлением тонуса детрузо$\mathrm{pa}$, рефлекторной возбудимости и порога чувствительности мочевого пузыря по результатам ретроградной цистометрии. Выявлено также отчетливое положительное влияние лазерного излучения на величину объемной скорости кровотока верхнепузырной артерии, что, по-видимому, лежит в основе его антигипоксического действия и, в целом, улучшает биоэнергетику детрузора мочевого пузыря $[15,17]$.

Одним из эффективных методов лечения гиперактивного мочевого пузыря является теплолечение (озокеритовые, парафиновые, грязевые аппликации) [18].

Лечебный эффект теплолечения связан с термическим, механическим (компрессионным) и химическим действием на организм ребенка. Химическое действие теплолечения обусловлено содержанием, главным образом в лечебных грязях и озокерите, минеральных солей, газов, микроэлементов, органических и биологически активных веществ, часть из которых может проникать через неповрежденную кожу. Это способствует репаративной регенерации, рассасыванию воспалительных инфильтратов, стимуляции защитных сил организма. Аппликации озокерита, парафина на область трусиковой зоны (мочевой пузырь и крестцовый отдел позвоночника) проводят при температуре $42-48^{\circ} \mathrm{C}$, продолжительностью 15-20 мин, на курс лечения до 10-15 процедур [21].

В последние годы появилась возможность использования пакетированной лечебной грязи и ее препаратов (экстракт лечебной грязи с рапой, масляные растворы лечебной грязи, ректальные суппозитории) как в условиях санатория, так и во вне курортных условиях. Под влиянием грязевых ап- пликаций отмечается благоприятная динамика клинико-функциональных показателей мочевого пузыря. Аппликации лечебной грязи применяют более низких температур $-38-39^{\circ} \mathrm{C}$.

Отдаленные результаты грязелечения выявили уменьшение числа рецидивов в 3,4 раза у детей с сопутствующим циститом [22].

С целью повышения эффективности лечения НДМП, методы физиотерапии применяются в комплексе с массажем и лечебной физкультурой, направленной на укрепление мышц тазового дна, передней брюшной стенки. В последние годы с успехом применяется новый вид вибромассажа с помощью аппаратов системы ЭПС (эластичные псевдокипящие слои), позволяющий использовать большой диапазон частот механических колебаний и тепловой компонент. Проведение терапии ЭПС на аноректальную зону у детей с НДМП позволяет получить торможение сокращений мочевого пузыря через систему детрузор-стабилизирующих рефлексов, что является актуальным для детей с ГАМП [19, 22].

Сегодня в детской практике более широко применяется метод биологической обратной связи. С помощью специального оборудования и программного обеспечения пациент получает возможность видеть и/или слышать свои физиологические свойства, такие как электрическая активность мышц, головного мозга. Это создает условия для терапевтического восстановления, нормализации этих физиологических реакций путем переобучения. Удетей с ГАМП тренировка мышц анального, уретрального сфинктеров с помощью специальных датчиков позволяет предотвращать повышение внутрибрюшного давления [2, 23].

Таким образом, медицинская реабилитация детей с ГАМП включает широкий спектр физических факторов, направленных на совершенствование акта мочеиспускания на всех уровнях иннервации мочевого пузыря.

\section{Список литературы}

1. Вишневский Е.Л., Гельдт В.Г., Николаев С.Н. Диагностика и лечение дисфункции мочевого пузыря у детей раннего возраста // Детская хирургия.-2002.- № 3.- с. 48-54.

2. Вишневский Е.Л., Гусева Н.Б., Игнатьев Р.О. Способ лечения детей с нейрогенной дисфункцией мочевого пузыря при снижении активности позыва к мочеиспусканию методом биологически обратной связи. // Патент на изобретение № 2452531.2012

3. Dumoulin C., Hay-Smith J. Pelvic floor muscle training versus no treatment, or inactive control treatments, for urinary incontinence in women. Cochrane Database Syst Rev. 2010; 20: 1. https:// www.ncbi.nlm.nih.gov/pubmed/20091581 
4. Меновщикова Л.Б., Николаев С.Н., Хан М.А., Гусева Н.Б. Корсунский А.А. Основные принципы диагностики и лечения расстройств мочеиспускания у детей и подростков: /Методические рекомендации № 10.- Медицина 2013.- c. 38

5. Любарская Ю.О., Атдуев В.А. Гиперактивный мочевой пузырь // Ремедиум Приволжье.- 2014.- № 5 - с. 36-41. https:// socionet.ru/publication.xml?h=spz: cyberleninka:33149:169058863.

6. Пушкарь Д.Ю., Аляев Ю.Г., Гльббочк П.В. Урология. / Российские клинические рекомендации.- М.: ГЭОТАРМедиа, 2014.-c.192 https:// www.ooorou.ru/public/uploads/ROU/Files/PКР-2017год.pdf

7. К Карпов Е.И. Лечение гиперактивного мочевого пузыря: расширяем горизонты// Медицинский совет.-2016.-№ 10c.140-142. https:// www.med-sovet.pro/jour/article/view/1417

8. Wu C., Franco I. Management of vesicoureteral reflux in neurogenic bladder. Investig Clin Urol. 2017 Jun;58(Suppl1):54-8. doi: 10.4111/icu.2017.58. S1. S54. Epub 2017 Jun 2.

9. Brownrigg N., Braga L., Rickard M., Farrokhyar F., Easterbrook B., Dekirmendjian A., Jegatheeswaran K., DeMaria J., Lorenzo A. The impact of a bladder training video versus standard urotherapy on quality of life of childrenwith bladder and bowel dysfunction: A randomized controlled trial. Int Urol Nephrol. 2017 Dec;49 (12):2105-9. doi: 10.1016/j. jpurol.2017.06.005.

10. Осипов И.Б., Сарычев С.А. Консервативное лечение нарушений резервуарной функции мочевого пузыря у детей с миелодисплазией// Педиатр.-2012.-т. 3.- № 4.- с. 67-73.

11. Интегративная урология: руководство для врачей /Под ред. П.В. Глыбочко, Ю.Г. Аляева.-М.: Медфорум,-2014.-c. 432

12. Меновщикова Л.Б., Хан М.А., Новикова Е.В. Физические методы в лечении гиперактивного мочевого пузыря. // Детская хирургия.-2008.-5.- c. 21-24

13. Costa Monteiro L.M., Cruz G.O., Fontes J.M., Vieira ETRC, Santos E.N., Araújo G.F., Ramos E.G.Rio de Janeiro R.J. Early treatment improves urodynamic prognosis in neurogenic voiding dysfunction: 20 years of experience. Jornal de Pediatria. July - August 2017; 93:4:420-7. doi: 10.1016/j.jped.2016.11.010

14. Гусева Н.Б. Длин В.В., Корсунский А.А. Ноктурия как симптом гиперактивного мочевого пузыря у детей. // Педиатрия.-2015.- т. 94.- № 3.-с.175-181.

15. Хан МА., Меновщикова Л.Б., Новикова Е.В. Медицинская реабилитация детей с нейрогенной дисфункцией мочевого пузыря.// Доктор. py.-2013.- № 10.-c.60-61. https:// elibrary.ru/item.asp?id=21218869

16. Syndrome of a hyperactive bladder. // Methodological recommendations of the DZM \#2. 2016;38 https:// www.ncbi.nlm. nih.gov/pmc/articles/PMC2600101/

17. Пушкарь Д. Ю., Касян Г.Р. Функциональная урология и уродинамика.-М: ГЭОТАР-Медиа,- 2013.- с. 376

18. Физиотерапия и курортология. Книга1. / Под редакцией В.М. Боголюбова. М.: Бином; - 2014.- с. 312

19. Новикова Е.В., Куянцева Л.В. Рассулова М.А., Трунова О.В. Современные технологии лазерного излучения в педиатрии. //Вопросы курортологии, физиотерапии и лечебной физической культуры.-2016; - 2 c. 119-120. https:// elibrary.ru/contents.asp?id=34419609

20. Kajbafzadeh A., Sharifi-Rad L., Ladi-Seyedian S., Mozafarpour S. Transcutaneous interferential electrical stimulation for the management of non-neuropathic underactive bladder in children: a randomised clinical trial. BJU Int. 2016 May;117 (5):793-800. doi: 10.1111/bju.13207.

21. Физиотерапия: национальное руководство / Под ред. Г.Н. Пономаренко - М.: ГЭОТАР-Медиа, 2013.- с. 864

22. Физическая и реабилитационная медицина: национальное руководство / Под ред. Г.Н. Пономаренко - М.: ГЭОТАР-Медиа, 2016.- с. 688

23. Синдром миелодисплазии у детей./ Под редакцией Николаева С.Н.-М.: Практическая медицина,-2018.- с. 320

\section{References}

1. Vishnevsky E.L., Geldt V.G., Nikolayev S.N. Diagnostics and treatment of bladder dysfunction in young children. Pediatric surgery. 2002; (3):48-54. (in Russ)

2. Vishnevsky E.L., Guseva N.B., Ignatiev R.O. The method of treatment of children with neurogenic dysfunction of the bladder with a decrease in the activity of urge to urinate by the method of biologically feedback. Patent for invention № 2452531.2012. (in Russ)

3. Dumoulin C., Hay-Smith J. Pelvic floor muscle training versus no treatment, or inactive control treatments, for urinary incontinence in women. Cochrane Database Syst. Rev. 2010; (20):1. https:// www.ncbi.nlm.nih.gov/pubmed/20091581 
4. Menovshchikova L.B., Nikolaev S.N., Khan M.A., Guseva N.B., Korsunsky A.A. The main principles of diagnosis and treatment of urination disorders in children and adolescents: Methodical recommendations № 10.- Medicine. 2013;38 (in Russ)

5. Lyubarskaya Yu.O., Atduev V.A. Hyperactive Bladder. Remedium Privolzhye. 2014; (5):36-41. (in Russ) https:// socionet. ru/publication.xml?h=spz: cyberleninka:33149:16905886

6. Pushkar'D. Yu., Alyaev Yu.G., Glybochko P.V. Urologiya. Rossijskie klinicheskie rekomendacii.- M.: GEHOTAR-Media, 2014; p.192 (in Russ)

7. Karpov E.I. Treatment of a hyperactive bladder: widening the horizons. Medical Council 2016; (10):140-2. (in Russ)

8. Wu C., Franco I. Management of vesicoureteral reflux in neurogenic bladder. Investig Clin Urol. 2017 Jun;58 (Supp11):54-8. doi: 10.4111/icu.2017.58. S1. S54. Epub 2017 Jun 2.

9. Brownrigg N., Braga L., Rickard M., Farrokhyar F., Easterbrook B., Dekirmendjian A., Jegatheeswaran K., DeMaria J., Lorenzo A. The impact of a bladder training video versus standard urotherapy on quality of life of childrenwith bladder and bowel dysfunction: A randomized controlled trial. Int Urol Nephrol. 2017 Dec;49 (12):2105-9. doi: 10.1016/j.jpurol.2017.06.005

10. Osipov I. B., Sarychev S.A. Conservative treatment of violations of the reservoir function of the bladder in children with myelodysplasia. Pediatric. 2012;3: (4):67-73. (in Russ)

11. Integrativnaya urologiya: rukovodstvo dlya vrachej. Pod red. P.V. Glybochko, YU. G. Alyaeva.- M.: Medforum, 2014;432 (in Russ)

12. Menovshchikova L.B., Han M.A., Novikova E. V. Fizicheskie metody v lechenii giperaktivnogo mochevogo puzyrya. Detskaya hirurgiya. 2008;5:.21-4 (in Russ)

13. Costa Monteiro L.M., Cruz G.O., Fontes J.M., Vieira ETRC, Santos E.N., Araújo G.F., Ramos E. G. Rio de Janeiro, $R . J$. Early treatment improves urodynamic prognosis in neurogenic voiding dysfunction: 20 years of experience. Jornal de Pediatria. July - August 2017; 93:4:420-7.

14. Guseva N.B. Dlin V.V., Korsunsky A.A. Knoturia as a symptom of a hyperactive bladder in children..Pediatria.2015;94:(3):175-181. (in Russ)

15. Khan M.A., Menovshchikova L.B., Novikova E. V. Medical rehabilitation of children with neurogenic dysfunction of the urinary bladder. Doctor. ru. 2013; (10):60-1. (in Russ) https:// elibrary.ru/item.asp?id=21218869

16. Syndrome of a hyperactive bladder. Methodological recommendations of the DZM \# 2. 2016;38. https:// www.ncbi.nlm. nih.gov/pmc/articles/PMC2600101/

17. Pushkar D. Yu., Kasyan G.R. Functional urology and urodynamics.-M: GEOTAR-Media, $2013 ; 376$ (in Russ)

18. Physiotherapy and balneology. Book 1. Edited by V.M. Bogolyubov. M.: Beenom; 2014;312 (in Russ)

19. Novikova E. V., Kuyantseva L.V. Modern technologies of laser radiation in pediatrics. Inquiries of balneology, physiotherapy and therapeutic physical training. 2016; (2):119-120 (in Russ)

20. Kajbafzadeh A., Sharifi-Rad L., Ladi-Seyedian S., Mozafarpour S. Transcutaneous interferential electrical stimulation for the management of non-neuropathic underactive bladder in children: a randomised clinical trial. BJU Int. 2016; May;117 (5):793-800.

21. National guidance on physiotherapy. Edited by Ponomarenko G.N.- PSP.: Peace and Family; 2008. (in Russ)

22. Physical and Rehabilitation Medicine: National Leadership. Ed. G. N. Ponomarenko. M. GEOTAR-Media; 2016. (in Russ)

23. Syndrome of myelodysplasia in children. Edited by Nikolaev S.N. M: Practical medicine, 2018;320. (in Russ)

\section{Авторы}

НОВИКОВА

Елена Владимировна

Elena V. NOVIKOVA
Кандидат медицинских наук, доцент, ведущий научный сотрудник отдела медицинской реабилитации детей и подростков ГАУЗ «Московский научно-практический центр медицинской реабилитации, восстановительной и спортивной медицины» Департамента здравоохранения г. Москвы. 105120, г. Москва, Земляной вал, д. 53; tel. +7(916)758-75-61, E-Mail: minkamal@mail.ru

Cand sci (Med), Associate Professor, Moscow Research Center for Medical Rehabilitation, Restorative and Sports Medicine of Moscow Health Department; Zemlyanoi val street 53, Moscow, Russia, 105120; tel. +7(916)758-75-61, E-Mail: minkamal@mail.ru 